\title{
Study on the application value of BACs-on-Beads technology combined with chromosome karyotype analysis in prenatal diagnosis
}

\author{
Lin Chen, Jianming Du, Junlong Wang, Shuangling Chen, Wei Wang, Wei Yang, Yanying Zhang, \\ Hui Zhang, Miao Zhang \\ Department of Laboratory Medicine, Harbin Red Cross Central Hospital, Harbin, China \\ Contributions: (I) Conception and design: L Chen, J Du, J Wang; (II) Administrative support: W Yang, Y Zhang, H Zhang; (III) Provision of study \\ materials or patients: H Zhang, S Chen; (IV) Collection and assembly of data: M Zhang, L Chen, J Du; (V) Data analysis and interpretation: L Chen, \\ W Yang, S Chen, Y Zhang; (VI) Manuscript writing: All authors; (VII) Final approval of manuscript: All authors. \\ Correspondence to: Lin Chen. Department of Laboratory Medicine, Harbin Red Cross Central Hospital, 415 Xinyang Road, Harbin 150076, China. \\ Email: clyx0451@126.com.
}

\begin{abstract}
Background: Bacterial artificial chromosome (BAC) marker-microsphere identification/separation technique [BACs-on-Beads (BoBs)] not only has a high detection rate for major chromosomal changes, but also for the other 9 microdeletion syndromes. In this study, the application value of BoBs combined with karyotype detection in prenatal diagnosis was evaluated.

Methods: The amniotic fluid samples of 132 pregnant women with prenatal diagnosis indications in Harbin Red Cross Central Hospital from June 2018 to June 2019 were collected and subjected to the detection of BoBs and routine karyotyping.

Results: Among the 132 pregnant women's amniotic fluid samples, 30 cases were abnormal in BoBs detection, with a detection rate of $22.73 \%$, and 29 cases were abnormal in chromosome karyotype analysis, with a detection rate of $21.97 \%$. Among them, 1 case of DiGeorge Type I microdeletion syndrome BoBs was successfully detected. The karyotype analysis failed to detect the same syndrome; the total coincidence rate of two methods was $99.24 \%$, the positive coincidence rate was $100.00 \%$, and the negative coincidence rate was $99.03 \%$; the sensitivity, specificity and positive predictive value (PPV), and negative predictive value (NPV) of the chromosome karyotype analysis was $96.67 \%, 100 \%$, and $99.03 \%$, respectively; the accuracy, specificity, and PPV/NPV of BoBs detection were $100 \%$.

Conclusions: When BoBs technology is combined with chromosome karyotype analysis, it can increase the detection rate of fetal chromosomal abnormalities, which could provide a basis for clinical prevention and follow-up diagnosis and treatment.
\end{abstract}

Keywords: BACs-on-Beads technology (BoBs technology); chromosome karyotype; prenatal diagnosis; amniotic fluid; abnormal

Submitted Dec 05, 2021. Accepted for publication Feb 16, 2022.

doi: $10.21037 / \mathrm{tp}-22-16$

View this article at: https://dx.doi.org/10.21037/tp-22-16

\section{Introduction}

There is a large population in China, which is facing the important public health problem of neonatal birth defects. Along with the rapidly increasing number of geriatric pregnancies caused by the opening of the second- and third-child policy in China, neonatal birth defects are increasingly prevalent, and it is necessary to undertake predictive and diagnostic measures in the prenatal period to 
control the birth rate of newborns with birth defects, reduce the neonatal mortality rate, as well as to reduce the burden on families and society. Chromosomal abnormalities are an important cause of birth defects, for which karyotyping could be considered the gold standard for detecting the number and structure of fetal chromosomes (1). However, the karyotyping method of detecting defects is complex and time-consuming (involving cell culture, slice making, and others processes), and the microdeletions, microduplications of $<5 \mathrm{Mb}$, and other structural abnormalities cannot be detected. Chromosomal microarray analysis (CMA) identifies a genetic cause in an additional $12 \%$ to $15 \%$ of affected children, as compared with the current standard of karyotyping, leading to recommendations that microarray analysis become the first-tier test for such children (2). The bacterial artificial chromosome (BAC) markermicrosphere identification/separation technique [BACson-Beads (BoBs)] was rapidly promoted, which was one method of Chromosomal microarray. The BoBs technology is a molecular detection technique based on the principle of liquid-phase gene microarray with biotin-labeled samples, hybridized with reference DNA and solidified into fluorescence-encoded microspheres, which are detected by specific BACs DNA probes. Finally, the chromosome number and structure were detected by Luminex system for classification and signal detection (Luminex Corp., Austin, TX, USA). Studies have demonstrated (3) that the BoBs technique has a high detection rate for the major chromosomal changes $(13,18,21, \mathrm{X}$, and $\mathrm{Y})$ as well as 9 other microdeletion syndromes (Wolf-Hirschhorn, CriduChat, DiGeorge Type I and II, and so on), which is also the reason for the superior feedback of BoBs technique over fluorescence in situ hybridization (FISH), quantitative fluorescent polymerase chain reaction (QF-PCR), and ultrasound during pregnancy (4).

Microarray analysis for prenatal diagnosis has been evaluated in some studies involving women whose fetus had a high probability of having chromosome abnormalities, such as those resulting in structural anomalies (5-9) . These studies have shown the situation in other countries or regions, and are not being carried out in Northeastern Region, China, for more evidence-based clinical application of the technique, in this paper, the amniotic fluid BoBs test combined with karyotype analysis was performed in 132 pregnant women to observe the value of BoBs test application. We present the following article in accordance with the STARD reporting checklist (available at https:// tp.amegroups.com/article/view/10.21037/tp-22-16/rc).

\section{Methods}

\section{Participants}

Amniotic fluid samples from 17-33 weeks gestation (mean, $22.36 \pm 2.03$ weeks) were collected from June 2018 to June 2019 from 132 pregnant women aged 35-41 years old with a mean age of $37.03 \pm 1.20$ years. The inclusion criteria for pregnant women stipulated that they must have at least 1 of the following indications for invasive prenatal diagnosis (10): (I) advanced age (age $\geq 35$ years); (II) high risk for serologic screening; (III) high risk for noninvasive DNA prenatal testing; (IV) abnormal fetal ultrasound structure; (V) history of birth of chromosomally abnormal children; (VI) family history; and (VII) chromosomal structural abnormalities in 1 of the spouses. The exclusion criteria included pregnant women who did not receive two testing modalities or failed in one of the testing methods. All procedures performed in this study involving human participants were in accordance with the Declaration of Helsinki (as revised in 2013). The study was approved by the ethics committee of Harbin Red Cross Central Hospital \{No.2021[14]\}. All pregnant women provided their written informed consent.

\section{Study methods}

\section{Specimen collection}

The medical staff informed 132 pregnant women to empty their bladders as much as possible before the collection, and they maintained the supine position with assistance during the sample extraction. Afterwards, amniocentesis punctures were made through the abdomen to the amniotic cavity, and about $30 \mathrm{~mL}$ of clear amniotic fluid was extracted. Samples were divided into 20 and $10 \mathrm{~mL}$ for karyotype analysis and BoBs detection, respectively.

\section{Karyotype analysis}

For karyotype analysis, the amniotic fluid samples were centrifuged at 2,000 rpm for $10 \mathrm{~min}$, the supernatant was discarded and incubated, and then it was harvested after 8-9 days according to the culture results. Each sample was counted and analyzed for 20-30 mitosis phases, and 5 karyograms were collected, observed, and analyzed, followed by photographing and preservation. The number of karyotype analysis was increased if any abnormalities were detected. 


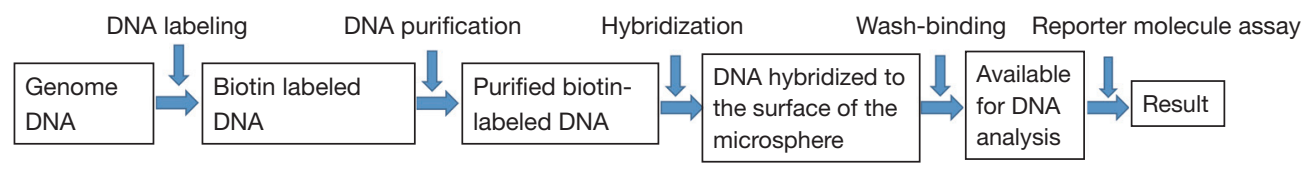

Figure 1 BoBs detection method. BoBs, BACs-on-Beads; BAC, bacterial artificial chromosome.

Table 1 Content of BoBs detection

\begin{tabular}{|c|c|c|}
\hline Target point & Chromosome region & Number of probes \\
\hline Chromosome aneuploidy 18 & $18 p 11.32-18 q 22.1$ & 5 \\
\hline Chromosome aneuploidy 21 & $21 q 22.11-21 q 22.3$ & 5 \\
\hline X chromosome aneuploidy & Xp22.31-Xq27.3 & 5 \\
\hline Wolf-Hirschhorn syndrome & $4 p 16.3$ & 5 \\
\hline CriduChat syndrome & $5 p 15.3-5 p 15.2$ & 8 \\
\hline Williams-Beuren syndrome & $7 q 11.2$ & 5 \\
\hline Langer-Giedion syndrome & $8 q 23-8 q 24$ & 7 \\
\hline Smith-Magenis syndrome & $17 \mathrm{p} 11.2$ & 4 \\
\hline DiGeorge Type I & $22 q 11.2$ & 4 \\
\hline DiGeorge Type II & $10 \mathrm{p} 14$ & 4 \\
\hline
\end{tabular}

BoBs, BACs-on-Beads; BAC, bacterial artificial chromosome.

\section{BoBs detection of amniotic fluid DNA extraction of samples}

Genomic DNA was extracted by column method by QIAamp DNA Blood Mini Kit, Qiagen (Germany) kit, and the concentration of DAN was determined by Hangzhou Allsheng Nano-100 microspectrophotometer after cell lysis and release, adsorption of DNA, removal of adhering proteins and ions, dissolution and other steps.

\section{Sample BoBs detection}

The genomic DNA of normal male and female controls, and samples was firstly labeled with enzymatic biotin nucleotides according to the instructions of Prenatal $\mathrm{BoBs}^{\mathrm{TM}}$ (Bacterial Artificial Chromosome LabelingMicrosphere Identification/Separation Method) (PerkinElmer, Waltham, MA, USA), and the labeled genomic DNA was subsequently purified. The purified labeled DNA and BoBs hybrid mesoporous microspheres mixture was run in a single well for overnight hybridization. Hybrid microspheres were washed with washing buffer, following which the microspheres were incubated with a reporter molecule (streptavidin-phycoerythrin) to bind the reporter molecule to the biotin-labeled genomic DNA. The microspheres were washed again to keep them suspended. The DNA fluorescence values bound to the microspheres were detected with the Luminex $200^{\mathrm{TM}}$ Cellular Assay System, data were collected, and the ratio of the sample detection value to the reference detection value was analyzed by BoBsoft 2.0 software (PerkinElmer). The method and the detection contents are shown in Figure 1 and Table 1.

\section{Statistical methods}

The statistical analysis of data was conducted using the 
Table 2 Confirmation rate of BoBs and chromosome karyotype

\begin{tabular}{lccc}
\hline Project & Number of cases & Confirmed BoBs, $\mathrm{n}(\%)$ & Chromosome karyotype analysis, $\mathrm{n}(\%)$ \\
\hline Confirmation rate & 132 & $30(22.73)$ & $29(21.97)$ \\
\hline
\end{tabular}

BoBs, BACs-on-Beads; BAC, bacterial artificial chromosome.

Table 3 Comparison of BoBs and chromosome karyotype analysis

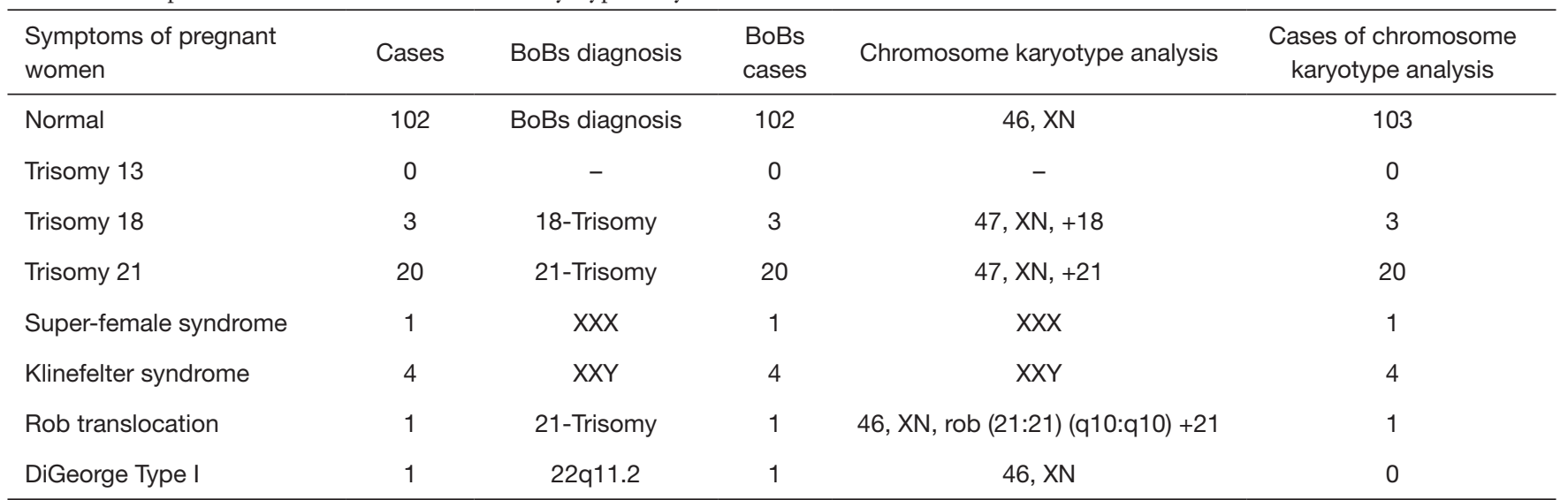

BoBs, BACs-on-Beads; BAC, bacterial artificial chromosome; Rob, Robertsonian translocation.

software SPSS 23.0 (IBM Corp., Armonk, NY, USA), and the main outcome analysis was the consistency of any numerical, structural, or submicroscopic chromosomal abnormalities between BoBs and conventional karyotype analysis, for which the categorical data were expressed as numbers and rate (\%).

\section{Results}

\section{Comparison of the detection results of two methods}

One hundred and thirty-two cases of amniotic fluid were detected successfully by BoBs and karyotype analysis, with a success rate of $100 \%$. Among 132 amniotic fluid samples from pregnant women with prenatal indications detected by BoBs, there were 30 abnormal cases, with a detection rate of $22.73 \%$; 29 abnormal cases of karyotype analysis, with a detection rate of $21.97 \%$, as shown in Table 2; a total of 0 cases of Trisomy 13, 3 cases of Trisomy 18, 20 cases of Trisomy 21, and 5 cases of sex chromosome abnormalities ( 1 case of XXX, 4 cases of XXY) was detected by two methods. One case of Robertsonian translocation (Rob) was detected by karyotype analysis as well as BoBs. One case of DiGeorge Type I microdeletion syndrome was successfully detected by BoBs, however, it was failed to be detected by karyotype analysis. Refer to Table 3.

\section{Comparison of the compliance rates of two assays}

Samples with normal results of two assays were included in the normal criteria, while the abnormal results of chromosome karyotype analysis or BoBs were included in the abnormal standard. Normal consistency was indicated by (-) and abnormal consistency was indicated by $(+)$. The overall compliance rate, the positive compliance rate, and the negative compliance rate was $99.24 \%, 100.00 \%$, and $99.03 \%$, respectively (Table 4).

\section{Comparison on sensitivity, specificity, positive predictive value (PPV), and negative predictive value (NPV) of two assays}

Specimens clearly diagnosed with chromosomal abnormalities were included as the "gold standard", and the sensitivity of conventional karyotype analysis was $96.67 \%$, the specificity and PPV were $100 \%$, and the NPV was $99.03 \%$. while the accuracy, specificity, and PPV/NPV of BoBs assay was $100 \%$, as shown in Tables 5,6. 


\section{Discussion}

With the continuous improvement of detection technology and the popularization of prenatal knowledge for pregnant women, the number of geriatric pregnant women receiving prenatal screening is gradually increasing and birth defects are gradually becoming a hotspot for research direction. Common karyotype analysis could detect chromosomal number abnormalities and structural abnormalities greater than $5 \mathrm{Mb}$. However, such analyses are time-consuming, have complicated operation, high culture limitation, and other defects. Moreover, some microdeletions/microduplications that can clearly lead to fetal malformations and abnormal intellectual development cannot be detected (11). For example, one case of DiGeorge Type I microdeletion syndrome in the study was missed in the detection of karyotype analysis (12).

Table 4 Comparison of compliance rate between BoBs and karyotype analysis

\begin{tabular}{lccc}
\hline \multirow{2}{*}{ BoBs } & \multicolumn{2}{l}{ Chromosome karyotype analysis } & \\
\cline { 2 - 3 } & + & - & \\
\hline+ & 29 & 1 & $100.00 \%(29 / 29)$ \\
- & 0 & 102 & $99.03 \%(102 / 103)$ \\
In total & 29 & 103 & $99.24 \%(131 / 132)$ \\
\hline
\end{tabular}

BoBs, BACs-on-Beads; BAC, bacterial artificial chromosome.
The current detection techniques for routine detection of microdeletions/microduplications are mainly CMA, secondgeneration sequencing (NGS), and BoBs techniques (13). Among them, CMA and NGS techniques detect pathogenic chromosome copy number abnormalities along with some undefined abnormalities of chromosome copy number, which could increase the difficulty of result interpretation and genetic counseling $(14,15)$.

The BoBs technique can compensate for these shortcomings. The basic principle is taken that a specific BAC can be hybridized with the genome of its target region, and the hybridized sample can be compared with the fluorescence intensity of the reference DNA and compared in fluorescence intensity, so that the changes of DNA copy number and structural defense could be determined and reflected. It has the advantages of no cell culture, high throughput, high speed, low quality of DNA requirement, clear results, easy interpretation, and others (16). In this study, 132 amniotic fluid samples of pregnant women with prenatal diagnostic indications were tested by BoBs combined with karyotype analysis, among which 103 cases were detected by karyotype analysis normally, 102 cases were detected by BoBs test normally, all detectable results could be yielded by BoBs tests $(22.73 \%, 30 / 132)$. While 1 case of karyotype analysis was missed $(21.97 \%, 29 / 132)$, the BoBs technique detected 29 fetal chromosomal number abnormalities and 1 chromosomal microdeletion without false positive results in the study, with higher success rate

Table 5 Comparison of the consistency between karyotype analysis and gold standard

\begin{tabular}{lcccccc}
\hline \multirow{2}{*}{$\begin{array}{l}\text { Chromosome karyotype } \\
\text { analysis }\end{array}$} & \multicolumn{2}{c}{ Gold standard } & Sensitivity & Specificity & PPV & NPV \\
\cline { 2 - 4 }+ & + & - & & & & \\
\hline+ & 29 & 0 & $96.67 \%(29 / 30)$ & $100.00 \%(102 / 102)$ & $100.00 \%(29 / 29)$ & $99.03 \%(102 / 103)$ \\
In total & 1 & 102 & - & - & - \\
\hline
\end{tabular}

PPV, positive predictive value; NPV, negative predictive value.

Table 6 Comparison of the consistency between BoBs and gold standard

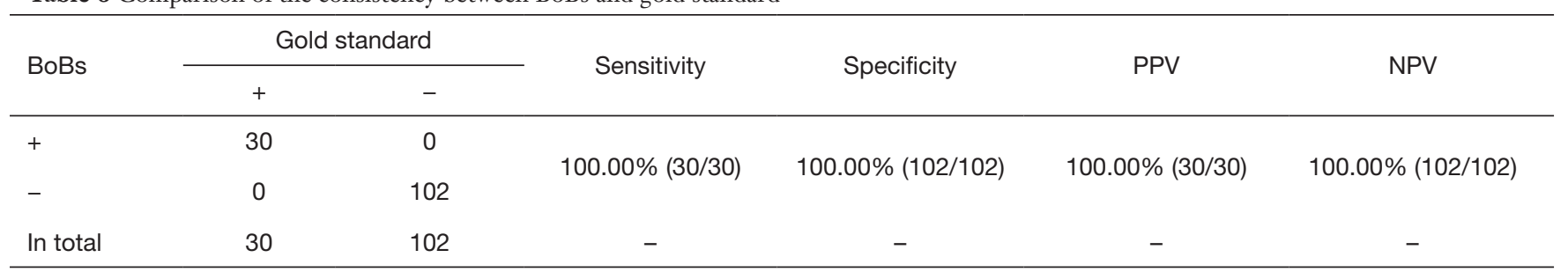

BoBs, BACs-on-Beads; BAC, bacterial artificial chromosome; PPV, positive predictive value; NPV, negative predictive value. 
and wider detection range. In further analysis, BoBs and karyotype analysis were consistent in detecting numerical abnormalities in aneuploidy, with 28 abnormalities detected (excluding 1 case of Robertson translocation and DiGeorge Type I microdeletion, respectively), with a detection rate of $21.21 \%$, with little difference compared with other studies $(17,18)$, which indicated the reliability of two tests for detecting aneuploidy abnormalities.

It should be noted that both tests were successful in detecting 1 case of karyotype analysis for Robertson translocation. However, 1 case of DiGeorge Type I microdeletion syndrome was successfully detected by BoBs. Fetuses with DiGeorge syndrome are more likely to have precocious heart disease, hypoparathyroidism, and mental retardation, for which it is usually $<5 \mathrm{Mb}$ after major fetal chromosomal aberrations, caused by the missed karyotype analysis. The presence of chromosomal microdeletion abnormality was confirmed by high-throughput genomic sequencing analysis. Therefore, the overall compliance rate of the two combined detection methods was $99.24 \%$ in the study, with a positive compliance rate of $100.00 \%$ and a negative compliance rate of $99.03 \%$.

Moreover, the sensitivity of the karyotype analysis was $96.67 \%$, the specificity and PPV were $100 \%$, and the NPV was $99.03 \%$; while the accuracy, specificity, and PPV/NPV with BoBs detection was $100 \%$, and the advantage of the assay data was significantly better, which was also caused by the missed detection of one microdeletion in karyotype analysis. Analysis suggested that it may be related to the fact that the BoBs technique reduces the detection steps for free of cultivation, and the chromosomal microdeletion syndrome often manifests as multiple malformations and growth retardation. The result fully demonstrates that the BoBs technique has certain advantages over karyotyping. Although the combination of both methods can improve the success rate of testing, it could help to shorten the detection cycle and reduce the decision-making time for pregnant women.

However, it should be noted that although BoBs assay can effectively compensate for the drawbacks of karyotype analysis and other assays, it also has some limitations, such as inability to detect ploidy changes, balanced rearrangements, point mutations, uniparental diploidy, methylation changes, chimerism, and others (19). The number of positive specimens was consistent with the number of positive cases. However, many studies have been reported in the literature, such as a study by Choy et al. (3) which reported that 7 cases of chimeric BoBs were not detected, while karyotype analysis had been detected successfully. It was relatively small sample size included in this study, with only 1 case of chromosomal microdeletion, which may have caused some bias in the results. Therefore, a larger sample size and conduction of an in-depth study is required in the future.

It could be concluded that the combination of karyotyping and BoBs testing can significantly improve the detection rate and reduce the risk of underdiagnosis, which could provide a more reliable basis for clinical follow-up.

\section{Acknowledgments}

Funding: This work was supported by the Applied Technology Research and Development Project in Heilongjiang Province (No. GZ19C02).

\section{Footnote}

Reporting Checklist: The authors have completed the STARD reporting checklist. Available at https://tp.amegroups.com/ article/view/10.21037/tp-22-16/rc

Data Sharing Statement: Available at https://tp.amegroups. com/article/view/10.21037/tp-22-16/dss

Conflicts of Interest: All authors have completed the ICMJE uniform disclosure form (available at https://tp.amegroups. com/article/view/10.21037/tp-22-16/coif). The authors have no conflicts of interest to declare.

Ethical Statement: The authors are accountable for all aspects of the work in ensuring that questions related to the accuracy or integrity of any part of the work are appropriately investigated and resolved. All procedures performed in this study involving human participants were in accordance with the Declaration of Helsinki (as revised in 2013). The study was approved by the ethics committee of Harbin Red Cross Central Hospital \{No.2021[14]\}. All pregnant women provided their written informed consent.

Open Access Statement: This is an Open Access article distributed in accordance with the Creative Commons Attribution-NonCommercial-NoDerivs 4.0 International License (CC BY-NC-ND 4.0), which permits the noncommercial replication and distribution of the article with the strict proviso that no changes or edits are made and the original work is properly cited (including links to both the 
formal publication through the relevant DOI and the license). See: https://creativecommons.org/licenses/by-nc-nd/4.0/.

\section{References}

1. Piotrowski K, Halec W, Wegrzynowski J, et al. Prenatal diagnosis of Langer-Giedion Syndrome confirmed by BACs-on-Beads technique. Ginekol Pol 2014;85:66-9.

2. Shaffer LG, Kashork CD, Saleki R, et al. Targeted genomic microarray analysis for identification of chromosome abnormalities in 1500 consecutive clinical cases. J Pediatr 2006;149:98-102.

3. Choy RK, Chen Y, Sun XF, et al. BACs-on-beads: a new robust and rapid detection method for prenatal diagnosis. Expert Rev Mol Diagn 2014;14:273-80.

4. Advani HV, Barrett AN, Evans MI, et al. Challenges in non-invasive prenatal screening for sub-chromosomal copy number variations using cell-free DNA. Prenat Diagn 2017;37:1067-75.

5. Hillman SC, Pretlove S, Coomarasamy A, et al. Additional information from array comparative genomic hybridization technology over conventional karyotyping in prenatal diagnosis: a systematic review and meta-analysis. Ultrasound Obstet Gynecol 2011;37:6-14.

6. Maya I, Davidov B, Gershovitz L, et al. Diagnostic utility of array-based comparative genomic hybridization (aCGH) in a prenatal setting. Prenat Diagn 2010;30:1131-7.

7. Srebniak M, Boter M, Oudesluijs G, et al. Application of SNP array for rapid prenatal diagnosis: implementation, genetic counselling and diagnostic flow. Eur J Hum Genet 2011;19:1230-7.

8. Park JH, Woo JH, Shim SH, et al. Application of a target array comparative genomic hybridization to prenatal diagnosis. BMC Med Genet 2010;11:102.

9. Faas BH, van der Burgt I, Kooper AJ, et al. Identification of clinically significant, submicroscopic chromosome alterations and UPD in fetuses with ultrasound anomalies using genome-wide 250k SNP array analysis. J Med Genet 2010;47:586-94.

10. Yang Y, Wang Y, Gao J, et al. Re-evaluation of indications

Cite this article as: Chen L, Du J, Wang J, Chen S, Wang W, Yang W, Zhang Y, Zhang H, Zhang M. Study on the application value of BACs-on-Beads technology combined with chromosome karyotype analysis in prenatal diagnosis. Transl Pediatr 2022;11(2):212-218. doi: 10.21037/tp-22-16 for prenatal diagnosis of advanced pregnant women under the new screening regime. Zhonghua Yi Xue Yi Chuan Xue Za Zhi 2020;37:1057-60.

11. Saldarriaga W, García-Perdomo HA, Arango-Pineda $\mathrm{J}$, et al. Karyotype versus genomic hybridization for the prenatal diagnosis of chromosomal abnormalities: a metaanalysis. Am J Obstet Gynecol 2015;212:330.e1-10.

12. Boormans EM, Birnie E, Oepkes D, et al. Comparison of multiplex ligation-dependent probe amplification and karyotyping in prenatal diagnosis. Obstet Gynecol 2010;115:297-303.

13. Miller DT, Adam MP, Aradhya S, et al. Consensus statement: chromosomal microarray is a first-tier clinical diagnostic test for individuals with developmental disabilities or congenital anomalies. Am J Hum Genet 2010;86:749-64.

14. Xia M, Yang X, Fu J, et al. Application of chromosome microarray analysis in prenatal diagnosis. BMC Pregnancy Childbirth 2020;20:696.

15. Xie X, Yu J, Qi Z, et al. Experience and lessons on guiding and governing clinical applications of chromosome microarray analysis in the United States. Zhonghua Yi Xue Yi Chuan Xue Za Zhi 2021;38:419-24.

16. Li C, Chen B, Zheng J, et al. Prenatal Diagnosis of BACson-Beads Assay in 3647 Cases of Amniotic Fluid Cells. Reprod Sci 2019;26:1005-12.

17. Fang Y, Wang G, Gu L, et al. Application of karyotype analysis combined with BACs-on-Beads for prenatal diagnosis. Exp Ther Med 2018;16:2895-900.

18. García-Herrero S, Campos-Galindo I, Martínez-Conejero JA, et al. BACs-on-Beads technology: a reliable test for rapid detection of aneuploidies and microdeletions in prenatal diagnosis. Biomed Res Int 2014;2014:590298.

19. Tao H, Shi J, Wang J, et al. Rapid prenatal aneuploidy detection of BACs-on-Beads assay in 4961 cases of amniotic fluid samples. J Matern Fetal Neonatal Med 2021;34:4090-6.

(English Language Editor: J. Jones) 